\title{
A Baseband-Matching-Resistor Noise-Canceling Receiver with a 3-Stage Inverter-Only OpAmp for High In-Band IIP3, and Wide IF Applications
}

\author{
Anoop Narayan Bhat, Student Member, IEEE, Ronan van der Zee, Member, IEEE, and Bram Nauta, Fellow, IEEE
}

\begin{abstract}
In this paper we propose a baseband noise-canceling receiver architecture to increase in-band linearity. A key feature of the architecture is that all active circuits are in baseband, including the LNTA. The LNTA operating at baseband frequencies allows the use of feedback to increase the linearity. The paper analyzes a trade-off that exists between in-band linearity and noise in mixer-first receivers and shows how the proposed architecture breaks such trade-off. The receiver targets high IF bandwidths, enabled by a TIA composed of an OpAmp using only inverters. The paper describes the stabilization mechanism of this OpAmp with a unity gain bandwidth (UGB) of 7.6GHz. The receiver is fabricated in $22 \mathrm{~nm}$ FDSOI CMOS. Measured results show an in-band IIP3 of $>9 \mathrm{dBm}$ for an IF bandwidth of $175 \mathrm{MHz}$ with sub-5dB NF across 1-6GHz LO.
\end{abstract}

Index Terms-Base-station, in-band linearity, noise-canceling, wide-band IF, inverters-only OpAmp, high UGB, stabilization, TIA, LNTA

\section{INTRODUCTION}

Recently, increasing in-band linearity has become an important focus in many sub- $10 \mathrm{GHz}$ receiver applications, mainly those where the band of interest may contain many signals, like cognitive radio [1], base station applications [2] and intra-band carrier aggregation scenarios [3]. Other emerging areas where high in-band linearity is necessary include self-interference cancellation for in-band full-duplex receivers with significant cancellation in the digital domain [4], [5] and MIMO applications involving beam-forming that takes place (partly) in the digital domain [6].

Most of the above applications are increasingly targeting higher IF-bandwidths, mainly driven by higher data-rate demands. For instance, [1] targets high IF-bandwidth for cognitive radio and $5 \mathrm{G}$ wireless applications. [7] also aims at high IF-bandwidth for $5 \mathrm{G}$ bands below $6 \mathrm{GHz}$. Similarly, works on base-station receiver designs [8], [9] have targeted high IF-bandwidth to support all 3GPP bands.

Therefore, receivers with both high in-band linearity and wide IF-bandwidth are desired for many applications. It is also desired that other receiver performances such as NF, Out of Band (OoB) linearity, and input matching are not degraded.

To achieve high linearity, mixer-first topologies have been popular, as they can postpone voltage swing to the end of the receive chain. A few of those architectures are shown in Fig. 1 .

Manuscript received MONTH 00, 0000; revised MONTH 00, 0000.

A. N. Bhat, R. A. R. van der Zee, and B. Nauta are with the IC Design Group, University of Twente, 7522 NB Enschede, The Netherlands. (e-mail: a.n.bhat@utwente.nl)
Receivers like in Fig. 1(a) ([10], [11]) generally rely on the impedance at the input of the baseband amplifier (-A in Fig. 1(a)) for input matching to achieve low NF. There will be significant swing at the input of the baseband amplifier in these receivers. Hence such receivers trade-off in-band linearity for low NF. The topology in Fig. 1(b) [2] is a good choice for high in-band linearity due to the virtual ground at the input of the TIA. However, it is more noisy due to the $50 \Omega$ matching resistor. The receiver shown in Fig. 1(c) [12] achieves virtual ground at the input of the TIAs and also cancels the noise of the matching resistor. However, the LNTA operating at RF frequencies either limits the input matching or the linearity. Fig. 1(d) [13] is another good choice to increase in-band linearity because of the virtual short between the inputs of the baseband amplifier. But due to its lack of input matching, it is not practical in many applications.

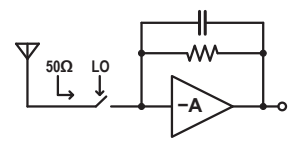

(a)

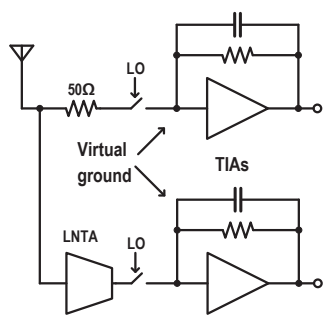

(c)

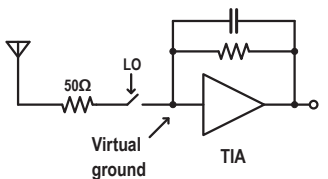

(b)

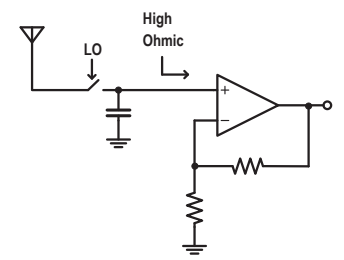

(d)
Fig. 1. Representative mixer-first receiver architectures (a) [10], (b) [2], (c) [12] , and (d) [13] for comparing their in-band linearity along with noise, matching, and OoB linearity performances

In [14], we proposed a Base Band Noise Canceling (BBNC) receiver architecture targeting both high in-band linearity and high IF-bandwidth without compromising on other performances such as NF, OoB linearity, and input matching. Compared to [14], in this paper we provide a more detailed analysis of the various properties of the architecture, as well as more mathematical analysis and design guidelines. We also provide analysis and design guidelines for the $7.6 \mathrm{GHz}$ unity gain bandwidth (UGB) 3-stage inverter-only OpAmp.

The rest of this paper is organized as follows. The proposed 
receiver architecture is presented and briefly described in section II. In depth analysis of the various properties of the architecture and trade-offs are given in section III. Section IV shows the full circuit implementation and circuit design details. This section also includes prior art on wide band TIA design and detailed analysis of the 3-stage inverter-only OpAmp. Section V deals with the experimental results and comparison with prior art. Section VI concludes the paper.

\section{Architecture}

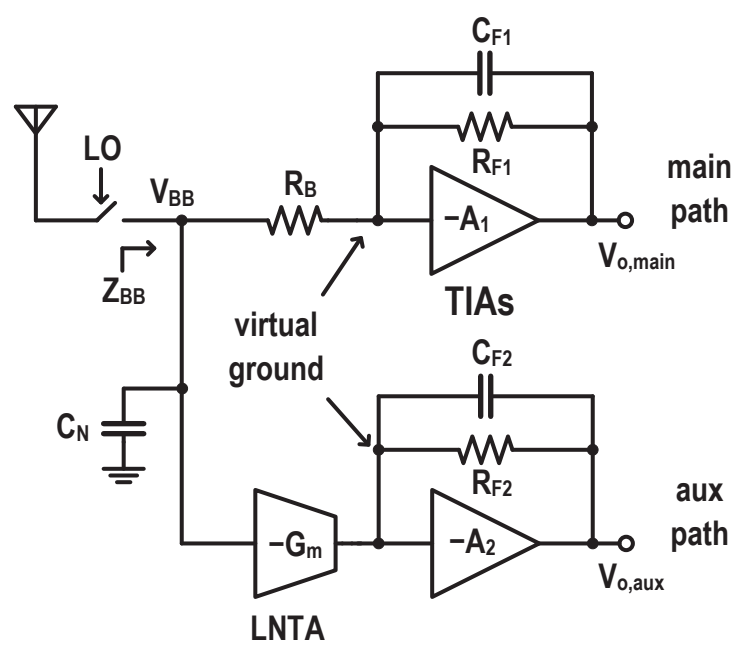

Fig. 2. Proposed baseband-matching-resistor noise-canceling receiver architecture

The proposed receiver is shown in Fig. 2. It is a noise-canceling architecture with the feature that all active circuits operate in baseband, including the LNTA. Input matching is provided by $R_{B}$, whose impedance is frequency translated to the input by the passive mixer. Both the TIAs have virtual ground at their input.

The proposed receiver achieves higher in-band linearity mainly because of the virtual ground at the input of the TIAs and the LNTA operating in baseband. The virtual ground at the input of the TIAs not only reduces the swing at their inputs, but also allows the loop-gain to be $>1$ unlike in the case of the architecture shown in Fig. 1 (a). Higher loop-gain further reduces the distortion produced by the TIAs.

Furthermore, operating the LNTA in baseband enables the use of feedback to achieve the desired linearity. Feedback not only increases the linearity of the LNTA, but also makes the linearity robust to PVT changes. Most RF LNTAs do not have this luxury, such that they dominate the overall non-linearity, with linearization techniques suffering from variation across PVT as explained in [15].

The N-path filter formed by the source impedance and the capacitor $C_{N}$ rejects OoB interferers. Unlike [12], where the LNTA operates at RF, input capacitance of the LNTA only (slightly) affect the bandwidth of the N-path filter and hence does not degrade input matching at high frequencies. Also, it is a noise canceling architecture where the noise of the matching resistor $R_{B}$ is canceled by an auxiliary path containing an LNTA with transconductance $G_{m}$. We analyze all the properties in greater detail in the next section.

\section{ARChitecture AnAlysis}

Fig. 3 shows a circuit model for mixer-first receivers mainly simplifying the frequency translation effects of the mixer switches in the receiver analysis [16], [11]. This model greatly simplifies analysis of input matching, noise, and conversion gain. Note that $Z_{i n}$ is the input impedance in the RF domain which has to be matched to $50 \Omega$. $Z_{B B}$ inside the dashed box is same as the $Z_{B B}$ in Fig. 2 which is the impedance towards the baseband part of the mixer switches. Similalry, the voltage $V_{B B}$ inside the dashed box represents the downconverted voltage on the baseband part of the mixer switches. The value of $\gamma$ in Fig. 3 depends on the number of paths and mixer switch duty cycle and is approximately 0.2 for the 4 -path, $25 \%$ we will use here. Similarly $4.3\left(R_{S}+R_{S W}\right)$ models the shunting impedance $\left(Z_{s h}\right.$ in [16]) for this case.

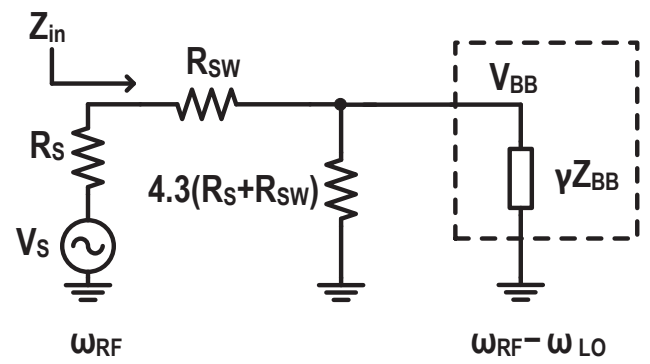

Fig. 3. A simplified circuit model for mixer-first receiver analysis [11], [16]

\section{A. Input Matching}

To simplify the input matching analysis, Fig. 3 can be rewritten as shown in Fig. 4 (a), considering only the resistive part $\left(R_{B B}\right)$ of the $Z_{B B}$. Since the input of the auxiliary path (LNTA) in Fig. 2 is capacitive and only visible out-of-band, only the main path is considered as shown in Fig. 4 (b) to calculate $R_{B B}$. The effect of the reactive part of $Z_{B B}$ is studied later in the subsection on N-path filtering and conversion gain. Also, the switch resistance $R_{S W}$ is neglected, since its value is designed to be low because its noise can not be canceled. Nevertheless, switch resistance is considered later for noise analysis.

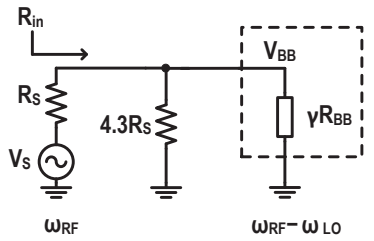

(a)

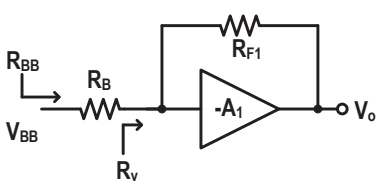

(b)
Fig. 4. (a) Circuit model for input matching, (b) circuit to calculate $R_{B B}$.

The analysis starts with the matching resistance $R_{i n}$ which should be equal to $50 \Omega$. Then $R_{B B}$ should be [17]:

$$
R_{B B}=\frac{4.3}{3.3} \times \frac{R_{s}}{\gamma}
$$

where $R_{S}$ is $50 \Omega$ and $\gamma$ is approximately 0.2 for 4-path filtering. Also, from Fig. 4 (b), $R_{B B}$ can be written as 


$$
R_{B B}=R_{B}+R_{v}
$$

where

$$
R_{v}=\frac{R_{F 1}}{1+A_{1}}
$$

Thus, from (2), it is a design freedom how to distribute $R_{B B}$ between $R_{B}$ and $R_{v}$.

Furthermore, from (3), it appears that for a chosen value of $R_{v}$, there are multiple sets of values of $R_{F 1}$ and $A_{1}$ available as design freedom. However, there is an additional design constraint coming from the required conversion gain from the antenna input to the output of the TIA which generally feeds the ADC. It can be seen from Fig. 4 (a) that the conversion gain from $V_{S}$ to $V_{B B}$ is a constant since $R_{B B}$ is fixed. If the gain from $V_{B B}$ to $V_{O}$ as shown in Fig. 4 (b) is fixed to $-A_{0}$, it can be written as

$$
\frac{V_{O}}{V_{B B}}=-\frac{R_{v} \times A_{1}}{R_{B}+R_{v}}=-A_{0}
$$

Since $R_{B}+R_{v}$ is fixed due to matching constraint (2), $R_{v} \times A_{1}$ also becomes fixed due to gain constraint (4). Hence only one of the $R_{B}, R_{v},-A_{1}$, or $R_{F 1}$ becomes an independent variable as varying one of them fixes the value of the others. $R_{v}$ is chosen here as the independent variable for the forthcoming analysis as it appears in all the three equations, i.e., (2), (3) and (4).

\section{B. In-Band Linearity}

The value of $R_{B B}$ can be calculated to be $321 \Omega$ from (1) for 4-path filtering. Hence $R_{v}$ can be varied from $321 \Omega$ to $0 \Omega$ according to (2) while varying $R_{B}$ by the same amount in the other direction. The equation to calculate noise factor from [17] is rewritten as follows to analyze the variation of NF when $R_{v}$ is varied from $321 \Omega$ to $0 \Omega$ :

$$
F=1+\frac{R_{S}}{4.3 \times R_{S}}+\frac{R_{B} \times R_{S}}{\gamma \times R_{B B}{ }^{2}}
$$

Note that to simplify the analysis, noise due to only $R_{B}$ of total $R_{B B}$ is considered in (5) as the noise due to $R_{v}$ (equivalently noise due to $R_{F 1}$ in Fig. 4 (b)) can be made negligible as illustrated in [17]. Also, $-A_{1}$ is assumed to be noiseless and the assumption that $R_{S W}$ is zero is continued.

Now, the variation in $R_{v}$ from $321 \Omega$ to $0 \Omega$ results in a NF variation from $0.9 \mathrm{~dB}$ to $3 \mathrm{~dB}$ as shown in Fig. 6. Hence most mixer-first architectures as in Fig. 1 (a) depend on achieving $R_{v}=321 \Omega$ to achieve low NF [11]. However, this is not the optimal choice for in-band linearity, as we will show next.

Consider the $I^{+}$slice of Fig. 5 (a) which is the main path of the architecture in Fig. 2. To understand the effect of different values of $R_{v}$ on in-band linearity, the loop gain $T_{0}$ (of the TIA with amplifier $-A_{1}$ ) will be formulated as a function of $R_{v}$. For this, $R_{E S}$ in Fig. 5 (a) is determined first.

$R_{E S}$ can be evaluated using the circuit in Fig. 5(b) following similar steps as in [2]. $R_{E S}$ is obtained from calculating the average current $I_{\text {test }}$ for the applied DC voltage $V_{\text {test }}$. The $\phi_{I^{+}}$represents the LO signal which controls the
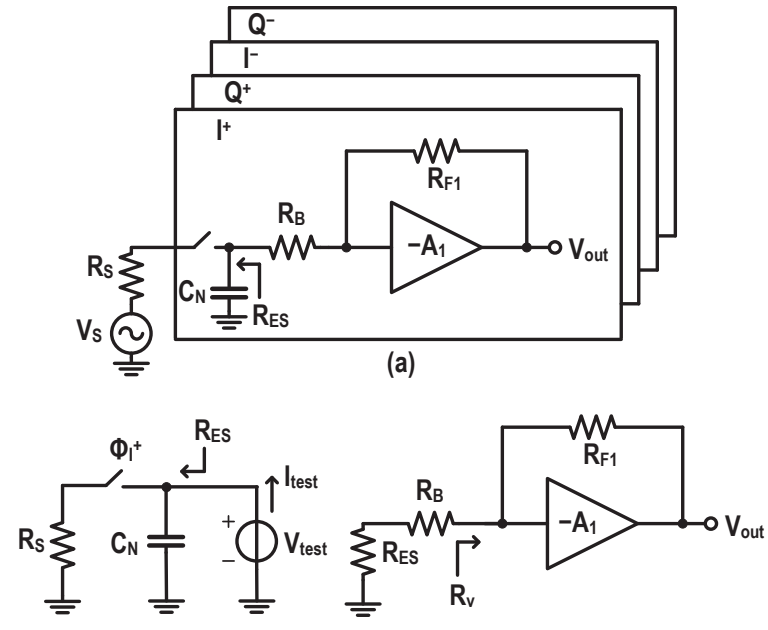

(b)

(c)

Fig. 5. (a) $I^{+}$slice of Fig. 2 to calculate $R_{E S}$, (b) equivalent circuit to evaluate $R_{E S}$ and (c) circuit model to analyze loop gain

mixer switch in the $I^{+}$path. Since the switching frequency of $\phi_{I^{+}}$and $-3 d B$ frequency due to $R_{S} C_{N}$ are both higher than the in-band frequencies of interest, $R_{E S}$ evaluated at DC can be used for all in-band frequencies. For $25 \%$ duty cycle $\phi_{I^{+}}$, average $I_{\text {test }}$ is equal to $V_{\text {test }} / 4 R_{s}$, resulting in $R_{E S}=200 \Omega$.

Now considering Fig. 5 (c), the loop gain $T_{0}$ of the TIA can be calculated as

$$
T_{0}=A_{1}\left(\frac{R_{E S}+R_{B}}{R_{E S}+R_{B}+R_{F 1}}\right)
$$

which can be rewritten using (2), (3) and (4) in terms of constants and the independent variable $R_{v}$ as

$$
T_{0}=\frac{A_{0} R_{B B}}{R_{v}} \times\left(\frac{R_{E S}+R_{B B}-R_{v}}{R_{E S}+R_{B B}+A_{0} R_{B B}}\right)
$$

Fig. 6 shows the graph of $T_{0}$ as a function of $R_{v}$ for $A_{0}=$ 10. It can be observed that $T_{0}$ increases with the decreasing of $R_{v}$. The higher the loop gain $T_{0}$, the higher the in-band linearity will be, since the coefficients ${ }^{1}$ of the nonlinear terms in the polynomial defining the nonlinear transfer function are suppressed by the loop gain $T_{0}$ [18], [19]. Thus a lower value of $R_{v}$ leads to higher in-band linearity. Note that this increase in in-band linearity generally comes at the cost of power since according to (4), gain $A_{1}$ has to be increased to obtain lower $R_{v}$.

Thus, a clear trade-off between NF and in-band linearity can be observed for the mixer-first architecture of the form in Fig. 1 (a), given the matching and conversion gain constraints. However, the noise canceling architecture proposed in Fig. 2 breaks this trade-off. Since high in-band linearity is targeted in this work, a low $R_{v}$ value is required which gives rise to degraded NF performance in the main path because all matching resistance must be provided by $R_{B}$. However, the auxiliary path cancels this noise, breaking the trade-off.

\footnotetext{
${ }^{1}$ The coefficients of the polynomial defining the nonlinear transfer function may also depend on the loop gain $T_{0}$ depending on the implementation of $-A_{1}$.
} 


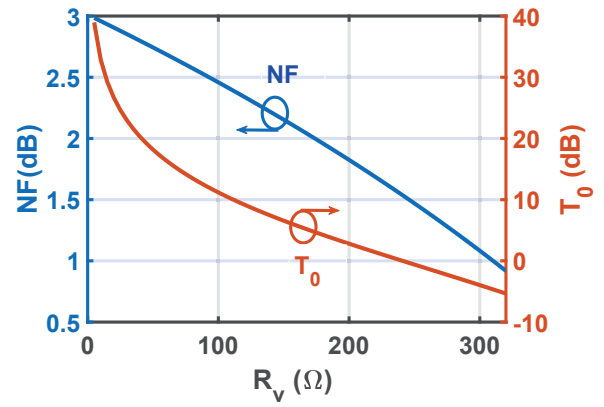

Fig. 6. NF and loop gain $T_{0}$ as a function of $R_{v}$.

The analysis above also holds for the TIA in the auxiliary path. However, since the output resistance of the LNTA is generally higher than the feedback resistance of the TIA, loop gain in the auxiliary path is $>1$ in most cases and hence linear.

The LNTA is another block that can limit the linearity of receivers especially LNTAs operating at RF frequencies such as the one in Fig. 1 (c). In section IV A about the circuit design we will show that because the LNTA can work in baseband in our topology, we can use more feedback for higher linearity.

\section{Noise}

Consider Fig. 7 to analyse the noise cancelling mechanism. Since the receiver is designed for high in-band linearity, virtual ground is assumed at the input of the TIAs. The noise voltage of matching resistor $R_{B}$ is represented by the voltage source $V_{n}$. Signal voltage and noise due to $V_{n}$ are pictorially represented at node $\mathrm{x}$ and the output of main and auxiliary path. It can be seen that the noise voltages at the output of both paths are in phase, while the signal voltages are out of phase. The condition for noise cancelling of the matching resistor $R_{B}$ is:

$$
V_{n} \times \frac{R_{F 1}}{R_{E S}+R_{B}}=V_{n} \times \frac{R_{E S}}{R_{E S}+R_{B}} \times \frac{R_{F 2}}{R_{L N T A}}
$$

which can be simplified to:

$$
\frac{R_{F 1}}{R_{E S}}=\frac{R_{F 2}}{R_{L N T A}}
$$

The different resistors in the above equation also impact various other specifications of the receiver. As calculated in the previous section, $R_{E S}$ is fixed by $R_{S}$. It will be shown in section IV A that $R_{L N T A}$ is chosen to obtain low noise in the LNTA. The resistor $R_{F 1}$ determines the gain of the main path. In this design, $R_{F 2}$ is adjusted to satisfy (9). The choice of $R_{F 2}$ also sets the gain of the auxiliary path. Note that $R_{E S}$ depends on external $R_{S}$ and does not follow the rest of the on-chip resistors in the above equation across PVT variations. However, to simplify the design, calibration circuits are not included since a significant noise cancellation is obtained even without such fine-tuning.

To include the effect of switch resistance $R_{S W}$ on noise, Fig. 4 (a) is rewritten as shown in Fig. 8 (a). The resistance $R_{B B}$ is approximated to $R_{B}$ in this model, since a low $R_{v}$

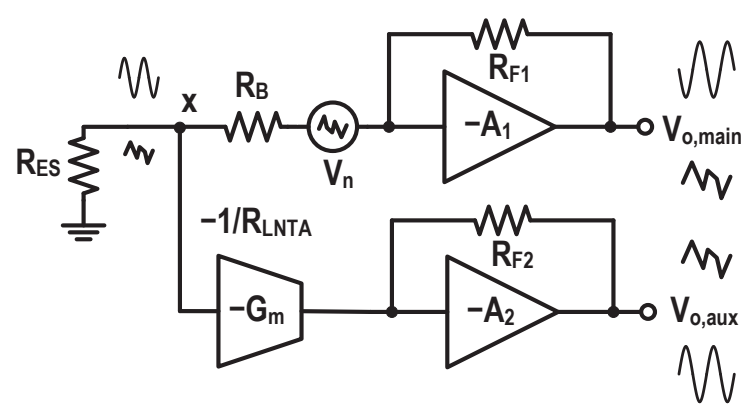

Fig. 7. Circuit to illustrate noise cancelling mechanism of $R_{B}$.

is chosen for high in-band linearity. Note that the $R_{B}$ also depends on $R_{S W}$ due to the input matching constraint:

$$
R_{B}=\frac{4.3}{\gamma} \frac{\left(R_{S}^{2}-R_{S W}^{2}\right)}{3.3 R_{S}+5.3 R_{S W}}
$$

Assuming that the noise due to $R_{B}$ can be cancelled, the effect of $R_{S W}$ on noise factor $F$ can be calculated from [17] as:

$$
F=\left(1+\frac{R_{S W}}{R_{S}}\right)\left(1+\frac{1}{4.3}\right)
$$

Fig. 8 (b) shows the plot of NF and $R_{B}$ for $R_{S W}$ between 0 and $50 \Omega$. It can be observed that NF increases from 0.9-3.9dB in this range. An $R_{S W}$ of $8 \Omega$ is chosen in this work. This value guarantees $<1.5 \mathrm{~dB} \mathrm{NF}$ when $R_{B}$ noise is cancelled. $R_{S W}$ is not decreased beyond this point as this would degrade input matching at higher frequencies due to parasitic capacitances. Moreover there are other sources of noise such as the LNTA and the TIAs.

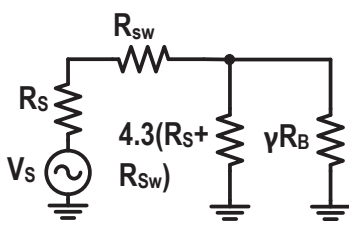

(a)

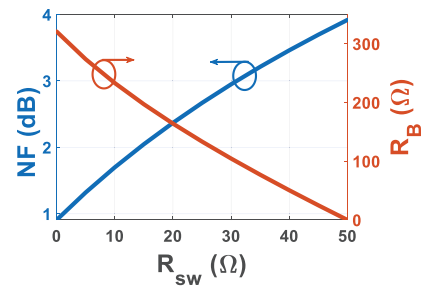

(b)
Fig. 8. (a) Circuit model and (b) plot to include $R_{S W}$ effect on NF and $R_{B}$.

\section{N-path Filtering and Conversion Gain}

The N-path filtering and conversion gain of Fig. 2 are analyzed as follows: first, the transfer function of the downconversion of the antenna voltage to $V_{B B}$ is calclulated and then the transfer functions from $V_{B B}$ to both the outputs $\left(V_{o, \text { main }}\right.$ and $\left.V_{o, \text { aux }}\right)$ are analyzed. Since the UGB of $-A_{1}$ is high, a virtual ground can be assumed at its input at near-out-of-band freqencies. Hence the $Z_{B B}$ in Fig. 2 is a parallel combination of $R_{B}$ and $C_{N}$.

Consider Fig. 3 to calculate $V_{B B}(s) / V_{S}$. For this, the voltage transfer from $V_{S}$ to the voltage across $\gamma Z_{B B}$ is determined first and then the resulting transfer is multiplied by 
$1 / \sqrt{4 \gamma}$ to include the downconversion effect [11]. To simplify the analysis, $R_{S W}$ is assumed to be zero, as a small $R_{S W}$ is chosen in the design and has little effect in this calculation.

Now, $2 \times V_{B B}(s) / V_{S}$ can be written as [11], [16]:

$$
\frac{V_{B B}(s)}{V_{S} / 2}=\frac{4.3 \sqrt{\gamma} R_{B}}{\left(4.3 R_{S}+5.3 \gamma R_{B}\right)\left(1+\frac{s(4.3 / 5.3) R_{S} R_{B} C_{N}}{(4.3 / 5.3) R_{S}+\gamma R_{B}}\right)}
$$

Since the above transfer function from RF source voltage to baseband side of the mixer switches is first order low pass in nature, the bandpass N-path filter formed at the RF side of the mixer switches is second order in nature. Note that the DC gain of (12) is $0.9 \mathrm{~dB}$ and the pole is a parallel combination of the resistors $R_{S}, 4.3 R_{S}, \gamma R_{B}$, and capacitor $C_{N} / \gamma$.

Now, again assuming virtual ground at the inputs of $-A_{1,2}$ (in Fig. 2) at near-out-of-band frequencies, it can be observed that the transfer functions from $V_{B B}$ to output of the main $\left(V_{o, \text { main }}\right)$ and auxiliary $\left(V_{o, \text { aux }}\right)$ paths are also first order low pass in nature with poles due to TIAs at $1 / R_{F 1} C_{F 1}$ and $1 / R_{F 2} C_{F 2}$ respectively.

Thus the overall conversion gain from RF voltage to output voltages of both main and auxiliary paths are second order low pass in nature. Also, note that both the poles, i.e., N-path pole and TIA pole are at real frequencies. In this work, to simplify the design, TIA poles are designed at $200 \mathrm{MHz}$ and the N-path filter pole is placed at sufficiently higher frequency of $475 \mathrm{MHz}$ such that $\mathrm{N}$-path filter pole does not strongly affect the overall IF bandwidth $(175 \mathrm{MHz})$ of the receiver.

\section{CiRCuit DESIGN AND IMPlementation}

Fig. 9 shows the circuit implementation of the proposed receiver in a $22 \mathrm{~nm}$ FDSOI CMOS process. An external balun with $1: \sqrt{2}$ turns ratio is used to convert the single ended RF input source to $100 \Omega$ differential input of the receiver. Mixer switches are driven by a $25 \%$ duty cycled clock operating at the LO frequency. On-chip amplifiers (to obtain square wave), $\div 2$ and $25 \%$ duty cycle generation circuits are used to generate such waveforms from a differential sinusoidal clock input operating at twice the LO frequency [20]. Fig. 9 also shows circuits to measure noise and linearity separately, which will be explained in the measurement section.

\section{A. LNTA}

As mentioned in the section on in-band linearity, LNTAs operating at RF frequencies can limit the linearity of the receivers. In this section, this limitation is explained and illustrated in the context of the source degenerated inverter used as LNTA in this work.

Consider the LNTA shown in Fig. 10 (a). For simplicity, let the matching be provided by the $R_{S}$ shown. The Value of $R_{L N T A}\left(2 \times R_{L N T A}=13 \Omega\right)$ is chosen such that noise due to $R_{L N T A}$ does not dominate the NF of the receiver. For a source degenerated inverter, the $g_{m} R_{L N T A}$ product indicates the amount of feedback, where $g_{m}=g_{m, M p}+$ $g_{m, M n}$ in the circuit considered. From [19], $P_{I I P 3}$ of the LNTA increases by increasing the $g_{m} R_{L N T A}$ product. In this simulation, $g_{m} R_{L N T A}$ is increased by impedance scaling the $g_{m}$, i.e., scaling the width of the transistors and current through the transistors together. Increasing $g_{m}$ also leads to higher capacitance at the input of the LNTA which will degrade input matching and lower the $-3 d B$ corner frequency $\left(\omega_{-3 d B}\right)$ for the transfer function $V_{i} / V_{s}$. Note that the effect of $C_{g s}$ of the input transistor on $\omega_{-3 d B}$ becomes constant with increase in $g_{m} R$ product due to the degeneration, however the effect of $C_{g d}$, which is approximately half of that of $C_{g s}$ in saturation region, increases.

Fig. 10 (b) shows $P_{I I P 3}$ and $\omega_{-3 d B}$ as a function of the $g_{m} R_{L N T A}$ product. The trade-off between $P_{I I P 3}$ and $\omega_{-3 d B}$ can be clearly observed. Hence operating LNTA in baseband as proposed in this work (Fig. 2) breaks this trade-off and allows for various feedback based architectures for LNTA to not only to increase linearity, but also make them robust across PVT which is not possible in architectures which does not have feedback [15].

The transistor length is chosen at $50 \mathrm{~nm}$ for the above simulation. The length is not decreased below this, even though a lower length improves $\omega_{-3 d B}$ for a given $g_{m} R_{L N T A}$ product as it also degrades the $P_{I I P 3}$ due to short channel length effects.

Fig. 9 shows the LNTA and its replica bias circuit implemented in this design. $2 \times R_{L N T A}$ is chosen to be $13 \Omega$ for low noise performance. A $g_{m}\left(g_{m, M p}+g_{m, M n}\right)$ value of $500 m S\left(g_{m} R_{L N T A} \approx 3.2\right)$ results in an IIP3 of $9 \mathrm{dBm}$. Note that $g_{m}$ is not increased beyond this point as this not only leads to higher power but also to higher supply voltage requirements. The receiver is powered by a single supply voltage of $0.83 \mathrm{~V}$.

The gates of the transistors $M p$ and $M n$ are biased with different voltages to increase the $g_{m} / I_{d d}$ and the $g_{m} / V_{d d}$ of the LNTA, where $I_{d d}$ and $V_{d d}$ refer to the DC current and supply voltage of the LNTA respectively. The $-3 d B$ corner frequency of the high pass filter formed by the AC coupling is $100 \mathrm{kHz}$. The replica bias circuit is a 12 times scaled down version of the LNTA. Output of the LNTA is biased around mid-supply, identical to the input of the TIA.

\section{B. TIA}

Designing for a wide IF-bandwidth in mixer-first receivers boils down to the design of a wide-band TIA. In mixer-first receivers where input matching is achieved by the feedback resistor of the TIA such as in [11], [10], stability is not a major concern since loop gain $T_{0}$ is less than 1 . However, in receiver architectures where the TIA needs to act as a virtual ground, $T_{0}$ greater than 1 is desired. Hence stability becomes a major challenge especially when wide IF bandwidth is also desired along with high in-band linearity. Therefore there is a revived interest in design of OpAmps with high UGB in such receiver applications.

Table I shows three recent works on such high UGB OpAmps. [21] utilizes pole-zero compensation to achieve high UGB. [21] achieves in-band IIP3 as high as $33 \mathrm{dBm}$, however note that the IF bandwidth is only $20 \mathrm{MHz}$ and it benefits from a $1.8 \mathrm{~V}$ supply. [1] and [22] employ feed forward based compensation to achieve high UGB. They report an in-band 


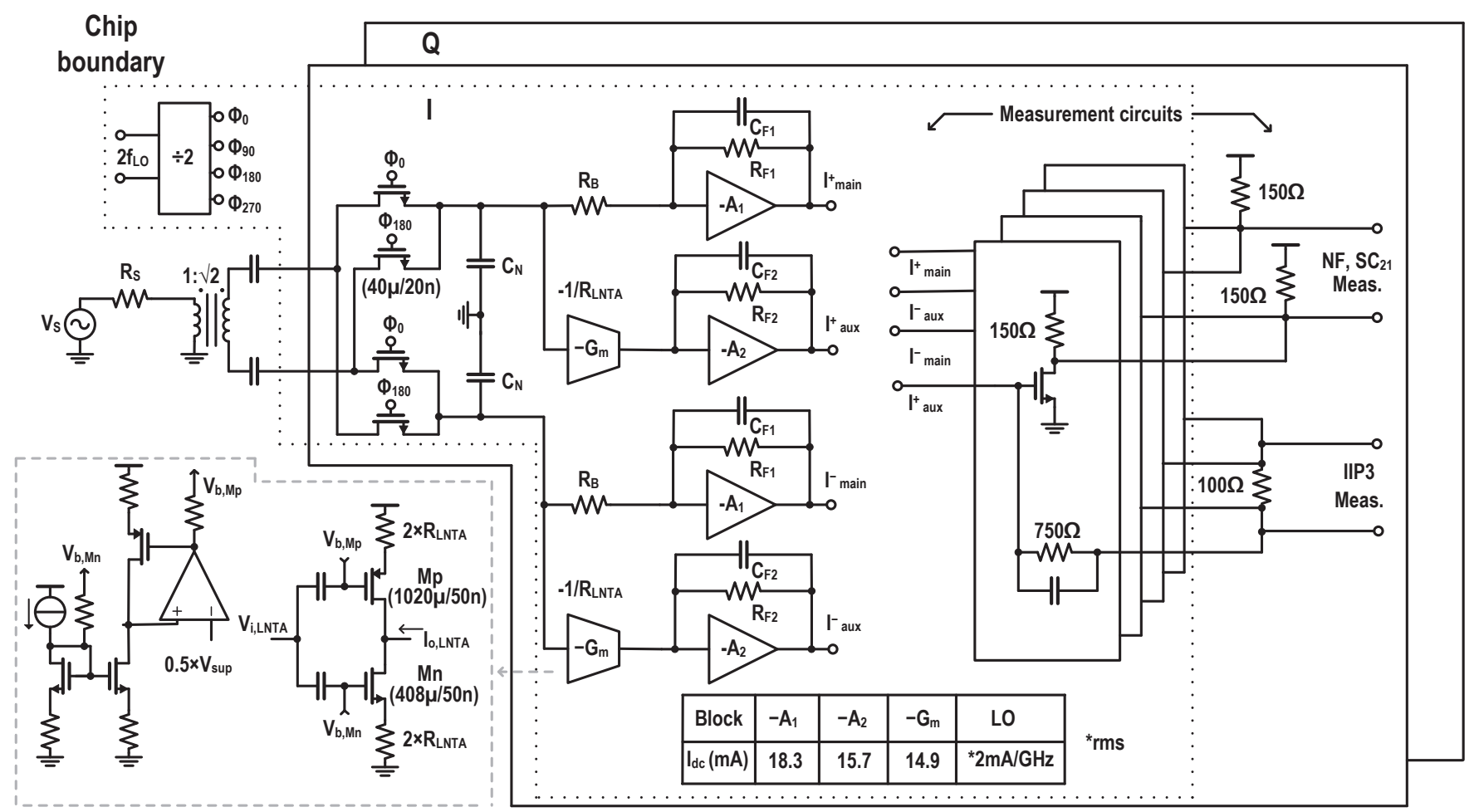

Fig. 9. Circuit implementation of the BBNC receiver.

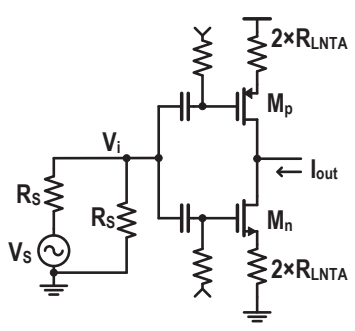

(a)

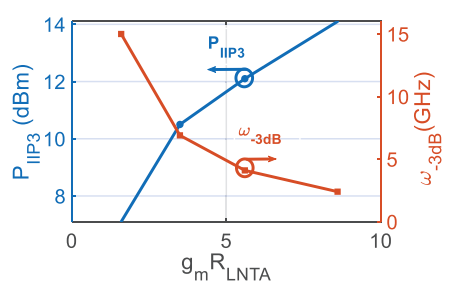

(b)
Fig. 10. (a) Simulation set-up of source degenerated LNTA operating at $\mathrm{RF}$ considered to demonstrate (b) trade off between $P_{I I P 3}$ and $\omega_{3 d B}$ as a function of $g_{m} R$ product.

IIP3 of $15.1 \mathrm{dBm}$ and $19.4 \mathrm{dBm}$ in the respective bandwidths of $200 \mathrm{MHz}$ and $80 \mathrm{MHz}$ and also benefit from a $1.8 \mathrm{~V}$ supply.

In our design, the OpAmps used in the TIAs of both main and auxiliary paths are designed using only inverters (DC coupled). First major advantage of inverter based analog design is that the design scales with the process, for example, inverter based OpAmp designed in this work uses the same supply $(0.83 \mathrm{~V})$ as that of digital circuits, whereas OpAmps designed in [1], [21], [22] and [3] needs a higher supply voltage compared to that used by the digital circuits. Secondly, the inverters avoid unnecessary internal nodes ([23]) such that the bandwidth can be high- this is reflected in the UGB reported in this work $(7.6 \mathrm{GHz})$. Other advantages include high SNR due to current reuse, and linearity benefits because of rail to rail output swing even though PSRR is poor. The result is an OpAmp with state-of-the-art performance that can work at this low supply voltage.

TABLE I

PERFORMANCE SUMMARY AND COMPARISON WITH THE PRIOR ART OPAMPS TARGETING WIDEBAND LINEAR TIA

\begin{tabular}{|c|c|c|c|c|c|}
\hline & Unit & $\begin{array}{c}\text { JSSC18 } \\
{[\mathbf{2 1}]}\end{array}$ & $\begin{array}{c}\text { TCASI19 } \\
{[\mathbf{1}]}\end{array}$ & $\begin{array}{c}\text { TCASI20 } \\
{[\mathbf{2 2}]}\end{array}$ & $\begin{array}{c}\text { This } \\
\text { Work }\end{array}$ \\
\hline Bandwidth & $\mathrm{MHz}$ & 20 & 200 & 80 & 175 \\
\hline $\begin{array}{c}\text { In-band } \\
\text { IIP3 }\end{array}$ & $\mathrm{dBm}$ & 33 & $15.1^{\ddagger}$ & 19.4 & $14^{\diamond}$ \\
\hline Supply & $\mathrm{V}$ & 1.8 & 1.8 & 1.8 & 0.83 \\
\hline UGB & $\mathrm{GHz}$ & 1.6 & 1.1 & 1.3 & 7.6 \\
\hline Noise $^{\dagger}$ & $\mu \mathrm{V}$ & 21.1 & $11.7^{\Delta}$ & 49.7 & 4.6 \\
\hline Power & $\mathrm{mW}$ & 5.4 & 18 & 14.2 & 13 \\
\hline
\end{tabular}

$\ddagger$ IIP3 of overall receiver

$\diamond$ Simulated overall receiver IIP3 with ideal LNTA block

$\dagger$ Input referred integrated (over respective IF bandwidth) noise of OpAmp

${ }^{\Delta}$ Integrating $0.83 \mathrm{nV} / \sqrt{\mathrm{Hz}}$ (at $10 \mathrm{MHz}$ ) over the IF bandwidth

The performance summary in Table $I$ is for the OpAmp in the auxiliary path. For this OpAmp, the feedback factor is one, because the output impedance of the LNTA is higher than the impedance of the feedback network. Analysis and design considerations can be extended to the OpAmp in the main path by considering the different feedback factor.

\section{3-Stage Inverter-Only OpAmp}

The stabilization mechanism and design considerations for the OpAmp are explained with the help of Fig. 11. For simplicity, no external load capacitor is assumed which had little effect on the stabilization technique or design 
(a)

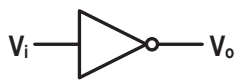

(b)

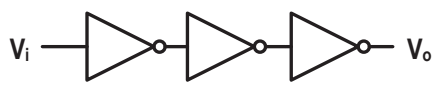

(c)

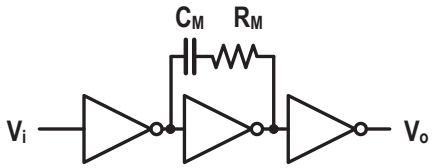

(d)

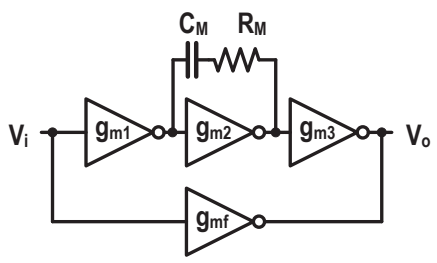

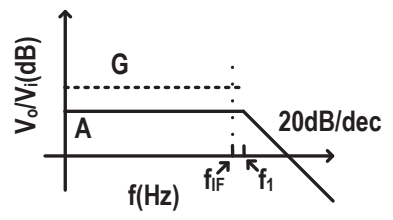
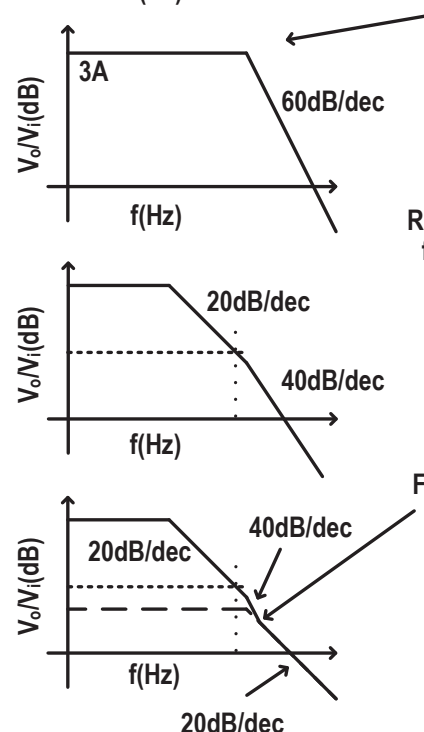

$20 \mathrm{~dB} / \mathrm{dec}$

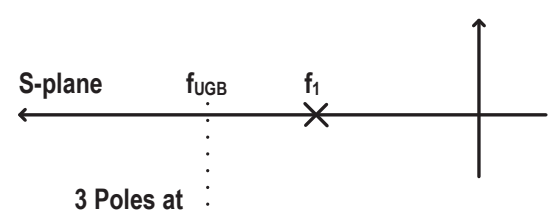

\begin{tabular}{|c|c|}
\hline$g_{\mathrm{mi}}$ & $\mathrm{mS}$ \\
\hline 1 & 104 \\
\hline 2 & 46 \\
\hline 3 & 94 \\
\hline $\mathrm{f}$ & 221 \\
\hline
\end{tabular}

\section{$R_{M}=22 \Omega$}

$\mathrm{C}_{\mathrm{M}}=5 \mathrm{pF}$

Fig. 11. Circuit, Bode plot and pole-zero plots to explain OpAmp Stabilization: (a) single stage inverter has insufficient self-gain, (b) 3-stage cascaded inverters roll-off at $-60 \mathrm{~dB} /$ decade through $\omega_{U G B}$, (c) Miller compensation with RHP zero removal improves roll-off at $\omega_{U G B}$ to $40 \mathrm{~dB} / \mathrm{dec}$ ade, still has potential for instability, and (d) a feed forward path introduces a zero and takes over high frequency response, hence $20 \mathrm{~dB} / \mathrm{decade}$ roll-off through $\omega_{U G B}$ and hence stabilizes OpAmp.

considerations as explained later in the section. The OpAmp needs to be designed for a certain loop gain $T_{0}$ to achieve the desired in-band linearity. Hence a minimum gain $\mathrm{G}$ is desired till the frequency $f_{I F}(\mathrm{~Hz})$ as shown in the dotted lines in the Bode plot of Fig. 11(a).

A simple choice is to use an inverter as the OpAmp. However, as shown in the Bode plot of Fig. 11(b), gain $A$ of the inverter is lower than the gain $G$ desired for linearity. Without loss of generality, the location of $f_{1}$, the pole frequency of the inverter is assumed to be at higher frequency than $f_{I F}$.

Since the overall OpAmp needs to be inverting, the next option in a single ended design is a cascade of three inverters, as shown in Fig. 11(b). Without loss of generality, each inverter stage is assumed to have a gain of $A \mathrm{~dB}$ and a single pole at frequency $f_{1}$ as shown in the corresponding Bode and pole-zero plots. The gain rolls-off with $60 \mathrm{~dB} /$ decade through the UGB, so the OpAmp is unstable when used in feedback.

As a first step towards stabilizing the 3-stage-inverter in Fig. 11(b), a Miller capacitor with Right Half Plane (RHP) zero removing resistor is added parallel to the middle inverter stage as shown in Fig. 11(c). Due to Miller compensation, the poles at input and output of the second stage inverters split into a dominant pole at frequency $f_{d}$ and a non dominant pole $f_{n d}$ which is pushed outside the UGB as shown in the pole-zero plot. The Miller capacitor value needs to be chosen such that the gain required at $f_{I F}$ (as shown in the dotted line of Bode plot) is sufficient to achieve the desired in-band linearity. $R_{M}$ is chosen such that the RHP zero is brought to the left half plane and is pushed far away from the UGB. However, note that circuit in Fig. 11(c) still has potential for instability as the magnitude in the Bode plot crosses the $f_{U G B}$ with $40 \mathrm{~dB} /$ decade roll-off due to $f_{d}$ and $f_{1}$ which reside below $f_{U G B}$.

A feed forward path as shown in Fig. 11(d) is added as a final step towards stabilization. The feed forward path adds a zero $^{2}$, so there are two poles and one zero below $f_{U G B}$. Hence the Bode plot goes through $f_{U G B}$ with $20 \mathrm{~dB} /$ decade roll-off as a first order system. The dashed line shown in the Bode plot is the magnitude response of the feed forward path. Overall magnitude response is dominated by the 3-stage cascaded inverter path till the zero is introduced and then the feed forward path takes over at high frequencies.

Fig. 11 shows the values of the $g_{m}$ of the inverters of various stages of the OpAmp. $g_{m 1}$ is chosen such that its thermal noise contribution is low. The length of the transistors of this stage is also chosen higher compared to other stages to reduce its flicker noise. $g_{m 2}$ is chosen to be lower compared to other stages to reduce the power as it does not affect the performance of the OpAmp significantly. $g_{m 3}$ is chosen such that it can source/sink sufficient linear current to the LNTA for the maximum input power level. $g_{m f}$ is chosen to adjust the feedforward zero location to obtain desired phase margin.

\footnotetext{
${ }^{2}$ The zero $Z_{f}$ due to feedforward path is located at higher frequencies than the $f_{I F}$ and does not severely degrade the settling characteristics of the OpAmp.
} 
The OpAmp is loaded by the input capacitance of the measurement circuits $(1.1 \mathrm{pF})$ as shown in Fig. 9. This pushes the pole at the output of $g_{m 3}$ to lower frequencies than $f_{1}$. In this design, this pole still remains higher than $f_{I F}$ as shown in Fig. 12 (a). Even though $f_{U G B}$ of the OpAmp is decreased due to the capacitive loading at its output, the gain of the OpAmp at $f_{I F}$ does not change.

Another practical consideration is that the feedback factor of the OpAmp is not exactly one and has a frequency dependence. This does not change the above analysis significantly as long as the output impedance of the LNTA is much higher than the impedance of the feedback network. However, this nonideality introduces an additional pole and zero in the loop gain. Fig. 12(b) shows the circuit model to include this effect. $-A_{2}$ in Fig. 12(b) is the OpAmp in the auxiliary path analyzed in Fig. 11. $R_{2}$ denotes the output resistance of the LNTA and $C_{2}$ represents the total capacitance at the output(input) of $\operatorname{LNTA}\left(-A_{2}\right)$. The transfer function of the feedback network can be written as:

$$
\frac{V_{2}(s)}{V_{1}(s)}=\frac{R_{2}}{R_{2}+R_{F 2}} \times \frac{1+s R_{F 2} C_{F 2}}{1+s\left(R_{F 2} \| R_{2}\right)\left(C_{F 2} \| C_{2}\right)}
$$

Thus, there will be one pole $\left(f_{f b}\right)$ and zero $\left(Z_{f b}\right)$ each in addition to the poles and zeros in the pole-zero plot of Fig. 11(d).

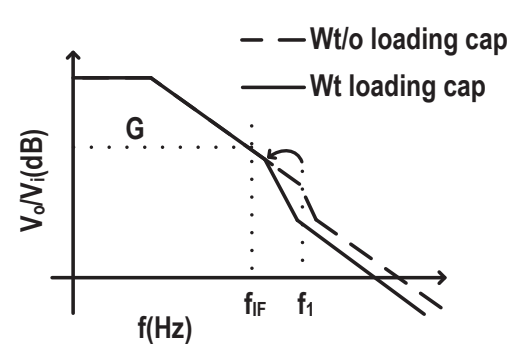

(a)

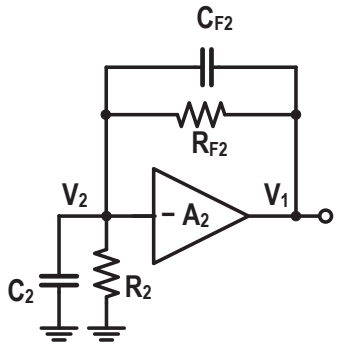

(b)
Fig. 12. (a) Bode plot showing the effect of capacitive loading at the output of the OpAmp and (b) Circuit to model pole and zero due to the feedback network.

Fig. 13 shows the simulation results of magnitude and phase response of the loop-gain $T_{0}$ of the OpAmp when placed in the TIA of the auxiliary path as shown in Fig. 9. The pole $\left(f_{f b}\right)$ and zero $\left(Z_{f b}\right)$ added by the feedback network along with $f_{1}$ and $Z_{f}$ are located between the dashed lines marked in the phase response of Fig. 13. It can be observed that $Z_{f b}$ at around $175 \mathrm{MHz}$ starts to improve the phase response, however, poles $f_{f b}$ and $f_{1}$ at slightly higher frequencies, start to degrade the phase response. However, feedforward zero $\left(Z_{f}\right)$ added at higher frequencies, improves the phase response again.

Fig. 13 also includes the stability plots for extreme conditions with respect to process and temperature along with nominal conditions. It can be observed that phase margin is $>75^{\circ}$ in all the cases. Location of the dominant pole due to Miller compensation can be seen at around $1 \mathrm{MHz}$ such that a loop gain of $38.2 \mathrm{~dB}$ is available at the band-edge to achieve the desired in-band linearity. The UGB is located at around $7.6 \mathrm{GHz}$ as marked in the simulation.
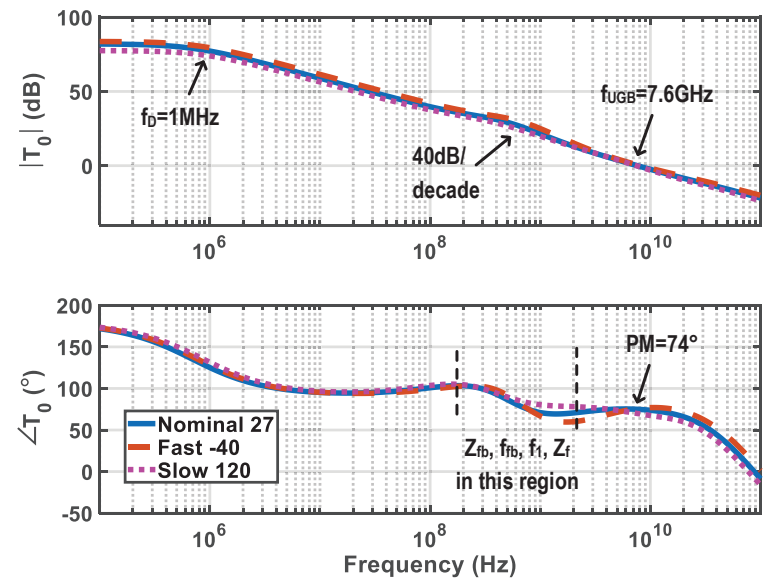

Fig. 13. Simulation of the magnitude and phase response of the Bode plot of the OpAmp designed and placed in auxiliary path of Fig. 9 with process corner results.

Since the UGB of the OpAmp is $7.6 \mathrm{GHz}$, high routing inductance during layout can lead to instability. Specifically, the routing inductance in the feedback path causes phase margin deterioration due to the series resonance of this inductance with $C_{F 2}$.

Fig. 14 shows the simulation results explaining this effect. The solid line with $74^{\circ}$ phase margin shows the stability plot of the OpAmp without considering routing inductors. The dashed line shows the stability plot when the feedback path with $R_{F 2}$ and $C_{F 2}$ is routed as a single path. The large dimension of $C_{F 2}(13.7 \mathrm{pF})$ in the feedback path results in a routing length of $200 \mu \mathrm{m}$ with equivalent routing indcutance of $200 \mathrm{pH}$ (assuming $1 \mathrm{nH} / \mathrm{mm}$ routing inductance). This results in a phase margin of $13^{\circ}$, hence potential for instability. Series resonance can be observed as a bump in the magnitude plot and a sharp phase degradation in the phase plot. Splitting the feedback path up in two parallel ones reduces the inductance and pushes the resonance to higher frequencies, improving the phase marigin to $72^{\circ}$ (dotted line).
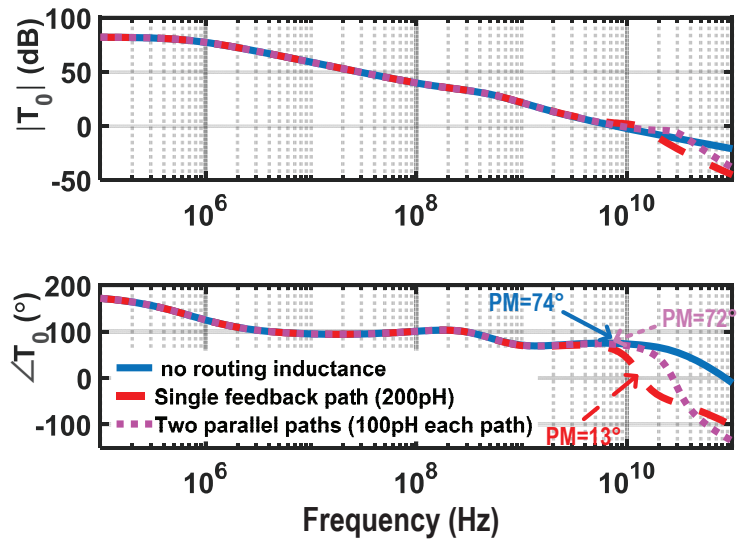

Fig. 14. Bode plot showing the stability degradation due to routing inductance. 
Note that common mode control is not necessary, since the OpAmps work independently and there is no coupling between any of the $\mathrm{I} / \mathrm{Q} /+/-/$ main/aux paths.

\section{Linearity, bandwidth, and power trade-off of the OpAmp}

The high in-band IIP3 and wide IF bandwidth targeted in this work leads to an increased power consumption. A trade-off between power and in-band IIP3 of the LNTA was discussed in section IV A. Here, first, the trade-off between the linearity and power consumption of the OpAmp is analyzed. The OpAmp in the auxiliary path is considered for the analysis and the same can be extended to the OpAmp in the main path.

In this analysis, the band-edge IIP3 of the OpAmp is simulated for various values of the loop gain $T_{0}$ (at the band-edge). For every $T_{0}$, the power consumption of the OpAmp is optimized. This gives a relation between the IIP3 and power consumption of the OpAmp. Note that the IIP3 of the OpAmp is characterized by simulating the IIP3 of the auxiliary path keeping the LNTA as an ideal block.

The dashed line in Fig. 15 (a) shows the simulation results of the IIP3 at the band-edge (175MHz) for varying values of $T_{0}$ (at the band-edge). The $T_{0}$ at the band-edge is varied as follows: first the miller capacitor is changed to change the $T_{0}$ at the band-edge. This results in a change in the stability conditions. Then the power consumptions in $g_{m 2}, g_{m 3}$, and $g_{m f}$ stages of the OpAmp are optimized such that the stability conditions are similar (constant phase margin) for all $T_{0}$. Note that $g_{m 1}$ is not varied such that thermal and flicker noise characteristics of the OpAmp are not varied significantly.

It can be observed from the solid line of the simulation result Fig. 15 (a) that a higher IIP3 (at higher $T_{0}$ ) at the band-edge comes at the cost of increased power consumption.

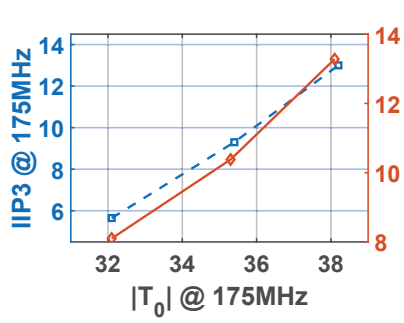

(a)

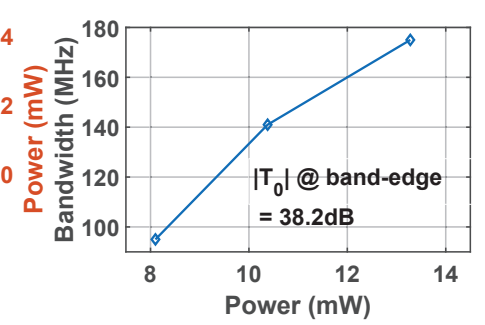

(b)
Fig. 15. Simulation results of the trade-off between the power and (a)band-edge IIP3, (b)bandwidth of the OpAmp

The trade-off between the bandwidth and power dissipation of the OpAmp can be analyzed with a similar simulation set-up with one key change. In the previous analysis, the IIP3 of the OpAmp is simulated against its power consumption $\left(T_{0}\right)$ at one frequency (band-edge). However, in this analysis, the frequency up to which $T_{0}>38.2 d B$ is valid is simulated against the power consumption of the OpAmp. This frequency indicates the IF bandwidth up to which a desired IIP3 can be obtained (as $T_{0}$ determines the IIP3). The simulation results in Fig. 15 (b) show that an increase in bandwidth also comes at the cost of the power consumption. A $T_{0}$ of $38.2 \mathrm{~dB}$ is chosen for this simulation since this value is used as $T_{0}$ in the final design.

\section{E. Power consumption breakdown}

TABLE II

POWER CONSUMPTION BREAKDOWN OF THE RECEIVER

\begin{tabular}{|c|c|c|c|c|c|}
\hline Block & $\begin{array}{c}\text { OpAmps } \\
\text { of main } \\
\text { path }\end{array}$ & $\begin{array}{c}\text { OpAmps } \\
\text { of Aux } \\
\text { path }\end{array}$ & LNTAs & LO & Total \\
\hline $\begin{array}{c}\text { Power } \\
\text { (mW) }\end{array}$ & $15.2 \times 4$ & $13 \times 4$ & $12.4 \times 4$ & $\begin{array}{c}1.7 \\
\mathrm{~mW} / \mathrm{GH} 2\end{array}$ & $\begin{array}{c}162.4 \mathrm{~mW} \\
+\mathrm{LO}\end{array}$ \\
\hline
\end{tabular}

Table II shows the breakdown of the power consumption of the receiver calculated using the current consumed by various blocks as shown in Fig. 9. The OpAmp of the main path consumes more power than the OpAmp of the auxiliary path. This is because the feedback factor of the main path is lower than that of the auxiliary path. Though a low $R_{S W}$ is required in the mixer switches for low noise, compared to baseband circuits, i.e., LNTA and TIAs, much lower power is dissipated in the LO generation circuits. This is because the mixer switches benefit from minimum length transistors $(20 \mathrm{~nm})$. The reduced capacitance for a given $R_{S W}$ and the reduced supply voltage due to lower transistor length greatly decreases the power dissipation in LO generation circuits compared to those designed with higher transistor length processes [11].

\section{EXPERIMENTAL RESULTS}

The receiver was realized on chip in a 22nm FDSOI CMOS process and has an active area of $0.48 \mathrm{~mm}^{2}$. A single supply voltage of $0.83 \mathrm{~V}$ powers the chip. Fig. 16 shows a chip photo with the placement of the various receiver blocks. Mixer switches are placed near to the bond-pad so that RF routing is minimal. The four capacitors involved in $\mathrm{N}$-path filtering are placed near the mixer switches to provide short return paths for the high frequency currents. The $c l k$ block includes a $\div 2$ circuit and a 4-phase $25 \%$ duty-cycle generation circuit.

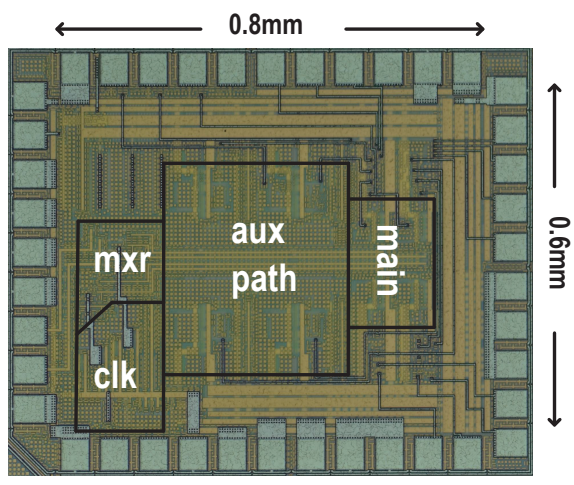

Fig. 16. Chip photo showing various receiver blocks

\section{A. Test Setup}

The interface of the receiver inputs and outputs for the measurement can be seen in Fig. 9. The measurements were performed with a single-ended source, followed by a passive balun driving the receiver. The differential output voltage is measured by an active differential probe. The 
receiver has circuits at the output to measure NF and IIP3 separately. Common-source amplifiers and all-pass voltage attenuator circuits are used at the output to measure NF and IIP3 respectively such that noise and distortion of the active differential probe do not dominate the respective measurements. The corresponding gain and attenuation were de-embedded. Note that Fig. 9 also shows the addition of the main path and auxiliary path signals.

\section{B. Measurement Results}

The measured IIP3 for both in-band and OoB are shown in Fig. 17. Two tones $f_{1}$ and $f_{2}$ are at $\Delta f-2 M H z$ and $\Delta f+$ $2 M H z$ respectively, for the case of in-band IIP3 measurement. Note that $\Delta f$ represents the offset from the LO frequency $\left(f_{L O}\right)$, and $f_{L O}=3 G H z$ in this case. IIP3 is $>9 \mathrm{dBm}$ for all $\Delta f$ within the measured TIA bandwidth of $175 \mathrm{MHz}$. For the OoB IIP3 measurement, two tones $f_{1}$ and $f_{2}$ are at $\Delta f$ and $2 \Delta f-50 \mathrm{MHz}$ such that the IM3 products are always at $50 \mathrm{MHz}$. An approximate increase of $6 \mathrm{~dB}$ can be observed for OoB IIP3 from $\Delta f$ of $500 \mathrm{MHz}$ to $1 \mathrm{GHz}$ indicating the effect of N-path filtering which has a $-3 \mathrm{~dB}$ frequency of $475 \mathrm{MHz}$. Since this $-3 d B$ frequency is more than two times away from the IF bandwidth, the advantage of OoB N-path filtering is not observed near band-edge. The measured IM3 curve for $\Delta f=20 \mathrm{MHz}$ is shown in Fig. 18 (a). This measurement shows that the IIP3 is valid till an input power of $-20 \mathrm{dBm}$ which is approximately $600 \mathrm{mVp}$-p swing at each single ended output.

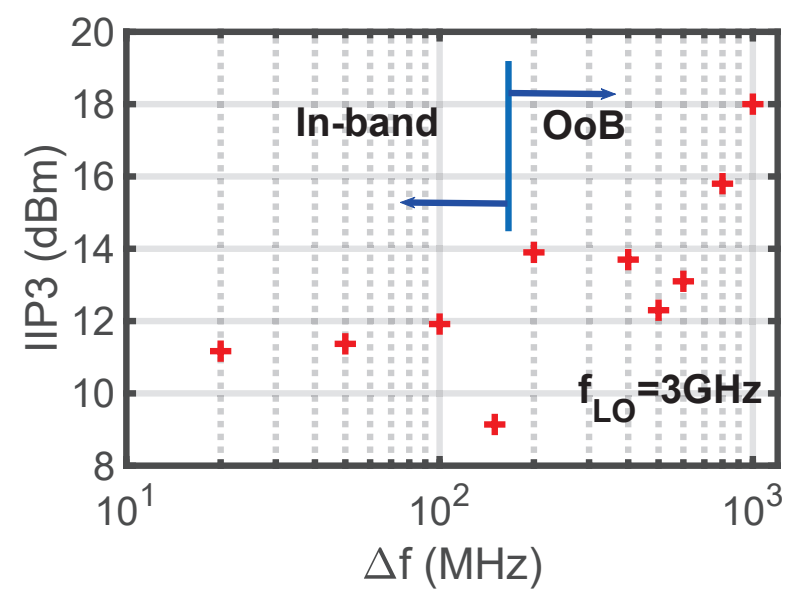

Fig. 17. Measured IIP3 across interferer offset frequencies

It can be recollected from section IV that the simulated in-band IIP3 of the LNTA is $9 \mathrm{dBm}$ and that of the receiver considering the LNTA as an ideal block is $14 \mathrm{dBm}$. Hence the overall in-band IIP3 of the receiver is limited by the LNTA. The measured in-band IIP3 of the receiver $(11 \mathrm{dBm})$ as shown in Fig. 17 is slightly higher than the IIP3 limited by the LNTA because of the more linear main-path that does not contain the LNTA. Although LNTAs such as [24] with more complex and stronger feedback can provide higher linearity, noise and power dissipation in such LNTAs increase due to the increased number of transistors. We use a source degenerated inverter as the LNTA as it provided the best trade-off among linearity, noise, and power of the circuits we considered.

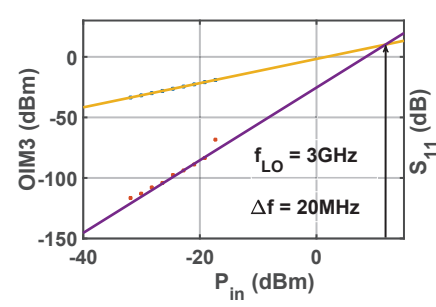

(a)

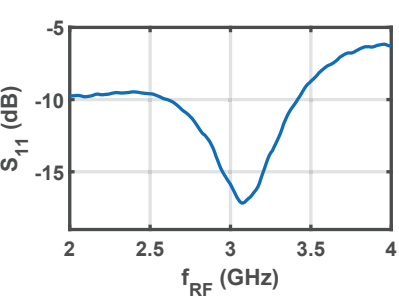

(b)
Fig. 18. Measured (a) OIM 3 at $20 \mathrm{MHz}$ offset and (b) $S_{11}$ showing N-path filtering

The $S_{11}$ measured at $f_{L O}=3 G H z$ is shown in Fig. 18 (b). Since the $-3 \mathrm{~dB}$ frequency of the N-path filter is more than two times higher than that of the IF-bandwidth, offset in $S_{11}$ due to parasitics such as bondwire inductance [25] [26] and mixer input capacitance does not degrade the input matching significantly. Fig. 19 shows the measured conversion gain $S C_{21}$ of the receiver. A dc-gain of $22.4 \mathrm{~dB}$ and $-3 \mathrm{~dB}$ bandwidth of $174 \mathrm{MHz}$ is measured which matches closely with the design and simulations.

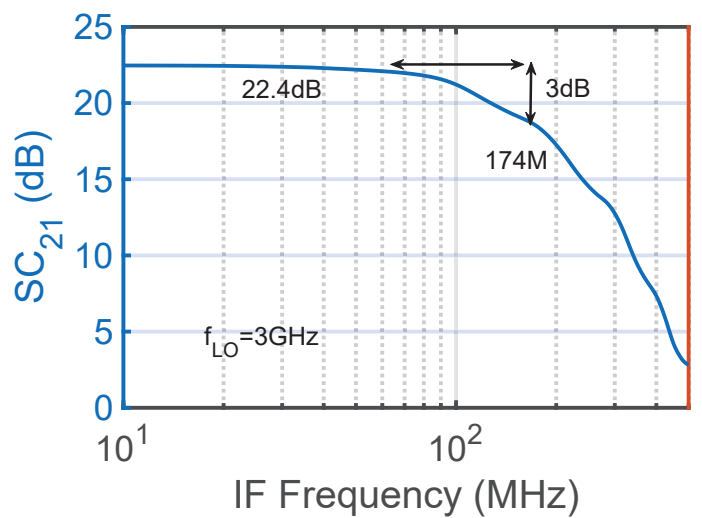

Fig. 19. Measured $S C_{21}$ at $3 \mathrm{GHz} \mathrm{LO}$

Fig. 20 shows the measured NF at $3 \mathrm{GHz}$ LO frequency. Simulation result shows a close match with the measurement. The $<3 \mathrm{~dB} \mathrm{NF}$ at $80 \mathrm{MHz}$ confirms the noise-canceling property of the receiver. For comparison, simulated NF of the receiver without noise-canceling path (only main-path) is also shown in Fig. 20. Note that since the IF-bandwidth is more than two times smaller than the $-3 d B$ frequency of the N-path filtering, the noise canceling is not affected by the N-path filtering across the IF bandwidth for a given LO frequency (3GHz in Fig. 20).

Fig. 21 shows the key performances of the receiver across LO frequencies. It plots the in-band IIP3, NF, and $S C_{21}$ measured at $50 \mathrm{MHz}$ offset across $\mathrm{LO}$ frequencies from 1-6GHz. In-band IIP3 stays between $9-11 \mathrm{dBm}$ for the measured LO sweep. The NF of $2.5 \mathrm{~dB}$ at $1 \mathrm{GHz}$ increases to around $5 \mathrm{~dB}$ at $6 \mathrm{GHz}$ LO. This degradation in NF is mainly due to increase in the effective mixer switch resistances at higher frequencies. Routing parasitics between the drivers and the mixer switches degrade the signal strength driving the mixer switches at higher frequencies and hence increase the 


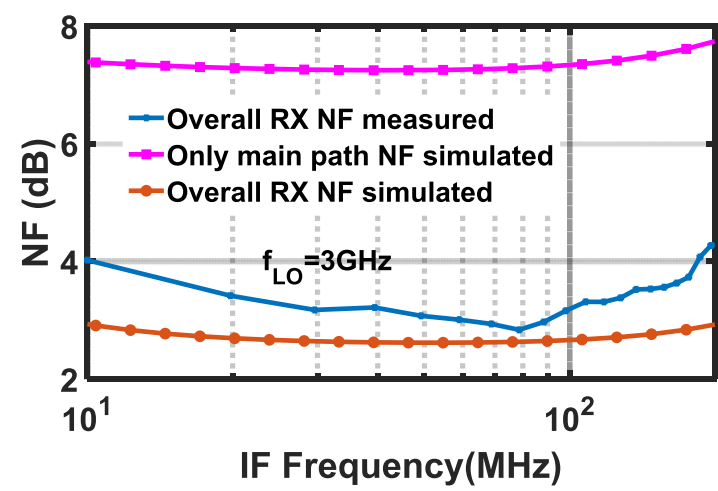

Fig. 20. Measured NF of the overall receiver at $3 \mathrm{GHz} \mathrm{LO}$.

effective mixer switch resistances. Increase in $R_{S W}$ not only degrades NF according to Fig. 8 but also increases $R_{E S}$ in noise canceling equation (9). Even though calibration to adjust the on-chip resistor values in (9) can improve the NF, such circuits are not included in this work. The $S_{11}$ well below $-10 \mathrm{~dB}$ across $1-6 \mathrm{GHz}$ shows that large input capacitances of the LNTAs does not degrade the input matching at higher frequencies, as explained in section IV A. The $S_{11}$ improves at higher $\mathrm{LO}$ frequencies compared to that at $2 \mathrm{GHz}$ due to the tuning effect of the mixer input capacitance by the series bondwire inductance [25] [26]. We measured the receiver till $6 \mathrm{GHz}$ even though it is functional till $8 \mathrm{GHz}$ in the extracted simulations due to the frequency limitation of the $2 \times f_{L O}$ source $(12.75 \mathrm{GHz})$ feeding the $c l k$ block.

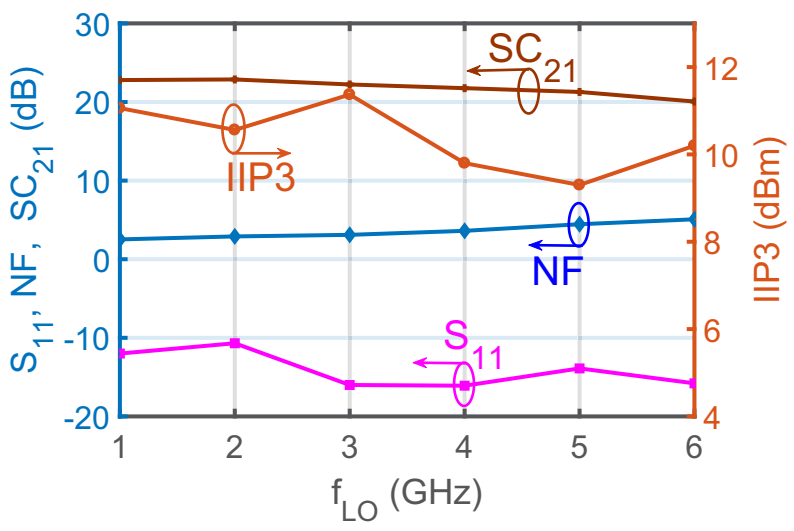

Fig. 21. Measured $S_{11}$, NF, $S C_{21}$, and IIP3 across LO frequencies

Fig. 22 shows the measured $C P_{1} d B$ (in-band) and $B_{1} d B$ (OoB). For the $C P_{1} d B$ measurement, input power at which the gain compresses by $1 \mathrm{~dB}$ is observed for various in-band frequency tones. For $B_{1} d B$ measurement, an in-band tone at $50 \mathrm{MHz}$ offset and $-25 \mathrm{dBm}$ input power is monitored for a $1 \mathrm{~dB}$ gain compression in the presence of OoB blocker tones at different frequency offsets.

Because of quasi-differential nature of the active building blocks, the receiver is characterized for its susceptibility to power supply and common mode noise. Fig. 23 shows the measured PSRR at different frequencies. A bias tee network is used to superimpose sinusoidal tones at various frequencies

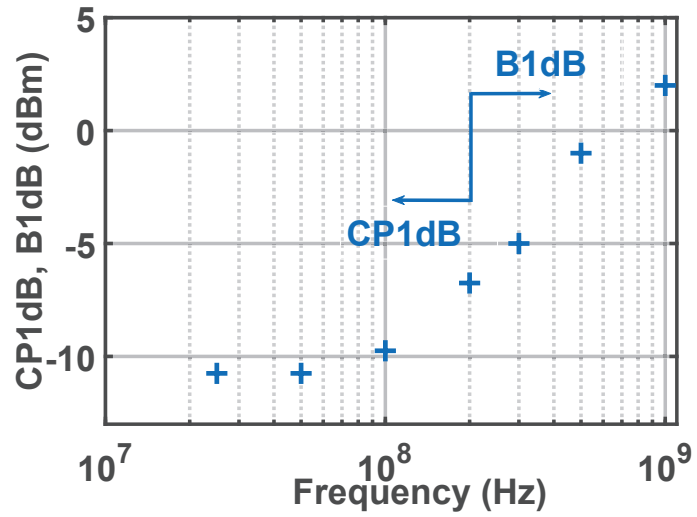

Fig. 22. Measured $C P_{1} d B$ and $B_{1} d B$ for various frequency offsets at $3 \mathrm{GHz}$ LO.

over the dc supply voltage across the off-chip decoupling capacitor placed close to the chip. A PSRR of around $38 \mathrm{~dB}$ is measured. Note that the voltage transfer from the off-chip decoupling capacitor to the on-chip decoupling capacitor decreases due to the increasing impedance of the bondwire inductance at higher frequencies. Therefore a higher PSRR is measured with respect to the off-chip decoupling capacitor above $100 \mathrm{MHz}$.

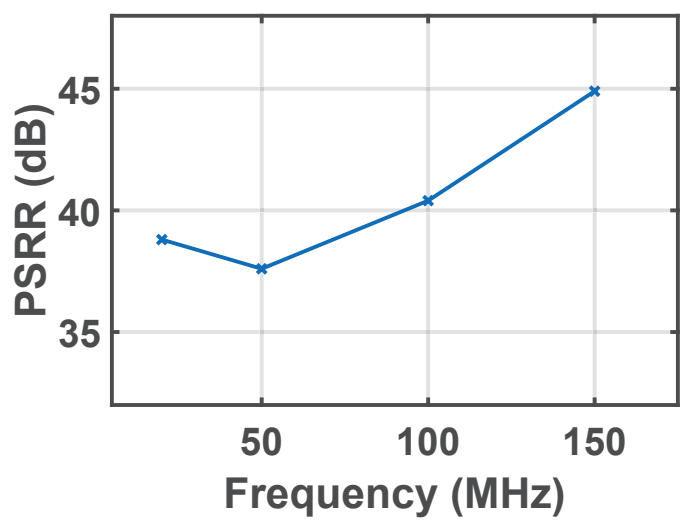

Fig. 23. Measured PSRR across IF frequency.

Thanks to the double balancing mixer used, the receiver rejects considerable common mode noise. However, unlike PSRR measurement where the supply-noise is fed to the singled-ended supply input of the chip at IF frequencies, the measurement accuracy of the CMRR is limited as the common mode noise is fed to the differential inputs of the chip at $\mathrm{RF}$ frequencies. An overall CMRR of $27 \mathrm{~dB}$ is measured at $3 \mathrm{GHz}$ LO limited by the low CMRR (35dB) of the balun and differential cables that feed the chip inputs. It is worth pointing out that even a mismatch of $0.25 \mathrm{~mm}$ between two bondwires that feed the receiver differential inputs can already degrade the CMRR to $27 \mathrm{~dB}$.

For similar reasons, i.e., mismatches and the limited CMRR, an in-band IIP2 of $33.9 \mathrm{dBm}$ is measured $\left(f_{1}=\right.$ $70.1 \mathrm{MHz}, f_{2}=120.1 \mathrm{MHz}$, and $f_{L O}=3 \mathrm{GHz}$ ).

Therefore to achieve high CMRR, PSRR, and IIP2 in such quasi-differential realizations, symmetric layout and large 
TABLE III

RESULT SUMMARY AND COMPARISON WITH PRIOR ART

\begin{tabular}{|c|c|c|c|c|c|c|c|}
\hline & This Work & TCASI19[1] & JSSC16[3] & RFIC19[5] & JSSC12[12] & ISSCC16[8] & ISSCC18[9] \\
\hline Architecture & BBNC & $\begin{array}{c}\text { RX with } \\
\text { LNTA }\end{array}$ & Mixer-first & Mixer-first & FTNC & ZIF RX & ZIF RX \\
\hline Technology & 22 FDX & $40 \mathrm{~nm}$ & $28 \mathrm{~nm}$ & $65 \mathrm{~nm}$ & $40 \mathrm{~nm}$ & $45 \mathrm{~nm}$ & $65 \mathrm{~nm}$ \\
\hline $\begin{array}{c}\text { In-band IIP3 } \\
\text { (dBm) }\end{array}$ & 9 & $15.1-16.7$ & $7^{*}$ & 6 & $-3^{\ddagger}$ & $-4^{\ddagger}$ & $12^{\dagger \ddagger}$ \\
\hline $\begin{array}{c}\text { IF bandwidth } \\
\text { (MHz) }\end{array}$ & 175 & 200 & 50 & 10 & 2 & 50 & 100 \\
\hline$f_{R F}(\mathbf{G H z})$ & $1-6$ & $3-6$ & $0.4-3.5$ & $0.5-3.5$ & $0.08-2.7$ & $0.4-4$ & $0.4-6$ \\
\hline NF (dB) & $2.5-5$ & $5-5.8$ & $2.4-2.6$ & 3.3 & 1.9 & 2 & 12 \\
\hline $\begin{array}{c}\text { Supply } \\
\text { Voltage (V) }\end{array}$ & 0.83 & $1.8 / 1.1$ & $1.5 / 1.1$ & $2.4 / 1.2$ & 1.3 & $2.5 / 1.8 / 1.1$ & $1.8 / 1.3$ \\
\hline Power (mW) & 172 & $64.1-69.6$ & $38-75$ & 38 & $35.1-78$ & 200 & $6600^{* *}$ \\
\hline Area $\left(\mathrm{mm}^{2}\right)$ & 0.48 & 1.2 & 0.23 & 1.5 & 1.2 & $49^{\star *}$ & $68.7^{\star \star}$ \\
\hline
\end{tabular}

${ }^{*}$ Measured for $20 \mathrm{MHz}$ bandwidth, baseband supply $=1.5 \mathrm{~V}$

$\dagger$ De-embedding off-chip LNA

$\checkmark$ Only I-channel

¥ taken from band-edge IIP3

$\triangle$ TDD operation mode

${ }^{\star \star}$ Full SOC

devices are needed to reduce mismatch. Common mode rejection circuits in quasi-differential implementations like in [23] can be investigated to further increase the above performances.

\section{Comparison}

Focus of this work is to achieve high in-band linearity and wide IF bandwidth without compromising on other receiver performances such as NF, OoB IIP3, matching, etc. Table III shows the in-band IIP3 and IF bandwidth (along with other performances) of the state of the art receivers and compares it to our receiver performance. [1] is a close comparison to our work as it also achieves both high in-band IIP3 and wide IF bandwidth. However, even though [1] improves NF by cross coupling the gates of a differential common gate LNTA, its NF is higher than $5 \mathrm{~dB}$, whereas we achieve much lower NF due to the noise-canceling property of the receiver. Also, [1] uses a higher supply voltage of $1.8 \mathrm{~V}$ to increase IIP3 compared to $0.83 \mathrm{~V}$ used in our receiver. Additionally, inductors used in the common gate LNTA not only limits their low-frequency operation $(3 \mathrm{GHz}$ compared $1 \mathrm{GHz}$ in our case) but also increases the chip area $\left(1.2 \mathrm{~mm}^{2}\right.$ for only I channel compared to $0.48 \mathrm{~mm}^{2}$ for both I and Q channels in our case). Nevertheless, due to the LNTA in the front-end, [1] does not possess the disadvantages of a mixer-first receiver [3], [27].

[3] and [5] are two other receivers that achieve high in-band IIP3, but both report this for a lower IF bandwidth of $20 \mathrm{MHz}$ and $10 \mathrm{MHz}$ respectively compared to $175 \mathrm{MHz}$ in our work. Furthermore they make use of higher analog supply voltages of $1.5 \mathrm{~V}$ and $2.4 \mathrm{~V}$ respectively to increase linearity. [9] measures a band-edge IIP3 of $12 \mathrm{dBm}$, but this is after de-embedding the off-chip LNA, which is reflected in the higher noise figure of $12 \mathrm{~dB}$. We mainly target base-station applications and our power numbers are lower compared to [8] and [9] which target the same application.

\section{CONCLUSION}

The proposed receiver can achieve high in-band linearity over a wide RF frequency range of $1-6 \mathrm{GHz}$. This is mainly because all active circuits operate at baseband frequencies and can be designed using feedback, both the LNTA and the TIA. The input capacitance of the LNTA in baseband does not degrade the input matching unlike that of an LNTA operating at RF frequencies. A strong feedback (high loop-gain) in the TIA in mixer-first receiver architectures increases their NF. The noise-canceling property of the proposed receiver breaks this trade-off allowing both low NF and high in-band linearity. A high loop-gain of the TIA with wide IF bandwidth also demands for an OpAmp with high UGB. An inverter-only multi-stage OpAmp is designed for this.

\section{ACKNOWLEDGEMENTS}

We would like to thank Texas Instruments for funding this project and Global Foundries for silicon donation. We thank Salvatore Finocchiaro and Francesco Dantoni for valuable discussions. We would like to thank H. de Vries and A. R. Rop for help during measurement and G. Wienk for CAD assistance.

\section{REFERENCES}

[1] J. Jiang, J. Kim, A. I. Karsilayan, and J. Silva-Martinez, "A 3-6-GHz highly linear I-channel receiver with over $+3.0-\mathrm{dbm}$ in-band $P_{1 d B}$ and 200-MHz baseband bandwidth suitable for $5 \mathrm{G}$ wireless and cognitive radio applications," IEEE Trans. Circuits Syst. I, Reg. Papers, vol. 66, pp. 3134-3147, Aug. 2019.

[2] D. Mahrof, E. Klumperink, Z. Ru, M. Alink, and B. Nauta, "Cancellation of opamp virtual ground imperfections by a negative conductance applied to improve RF receiver linearity," IEEE J. Solid-State Circuits, vol. 49, May 2014.

[3] C. Wu, Y. Wang, B. Nikolić, and C. Hull, "An interference-resilient wideband mixer-first receiver with LO leakage suppression and I/Q correlated orthogonal calibration," IEEE J. Solid-State Circuits, vol. 64, pp. 1088-1101, Apr. 2016. 
[4] D. Broek, E. Klumperink, and B. Nauta, "An in-band full-duplex radio receiver with a passive vector modulator downmixer for self-interference cancellation," IEEE J. Solid-State Circuits, vol. 50, pp. 3003-3014, Dec. 2015.

[5] A. Ershadi and K. Entesari, "A 0.5-to-3.5 GHz self-interference-canceling receiver for in-band full-duplex wireless," in IEEE Radio Freq. Integr. Circuits Symp., Jun. 2019, pp. 151-154.

[6] H. Krishnaswamy and L. Zhang, "Analog and RF interference mitigiation for integrated MIMO receiver arrays," Proc. IEEE, vol. 104, no. 3, pp. 561-575, Mar. 2016.

[7] G. Pini, D. Manstretta, and R. Castello, "Analysis and design of a 260-MHz RF bandwidth, +22-dBm OoB-IIP3 mixer-first receiver with third-order current-mode filtering TIA," IEEE J. Solid-State Circuits, vol. 55, pp. 1819-1829, Jul. 2020.

[8] N. Klemmer et al., "A 45nm CMOS RF-to-bits LTE/WCDMA FDD/TDD $2 \times 2$ MIMO base-station transceiver SoC with $200 \mathrm{MHz}$ RF bandwidth," in IEEE ISSCC Dig. Tech. Papers, Feb. 2016, pp. 164-165.

[9] D. McLaurin et al., "A highly reconfigurable 65nm CMOS RF-to-bits transceiver for full-band multicarrier TDD/FDD 2G/3G/4G/5G macro basestations," in IEEE ISSCC Dig. Tech. Papers, Feb. 2018, pp. 162-164.

[10] C. Andrews and A. Molnar, "A passive mixer-first receiver with digitally controlled and widely tunable RF interface," IEEE J. Solid-State Circuits, vol. 45, pp. 2696-2708, Dec. 2010.

[11] Y. Lien, E. Klumperink, B. Ternbroek, J. Strange, and B. Nauta, "Enhanced-selectivity high-linearity low-noise mixer-first receiver with complex pole pair due to capacitive positive feedback," IEEE $J$. Solid-State Circuits, vol. 53, pp. 1348-1360, May 2018.

[12] D. Murphy, H. Darabi, A. Abidi, A. Hafez, A. Mirzaei, M. Mikhemar, and M. Chang, "A blocker-tolerant, noise-cancelling receiver suitable for wideband wireless applications," IEEE J. Solid-State Circuits, vol. 47, pp. 2943-2963, Dec. 2012.

[13] M. Soer, E. Klumperink, Z. Ru, F. Vliet, and B. Nauta, "A 0.2-to-2GHZ $65 \mathrm{~nm}$ CMOS receiver without LNA achieving $>11 \mathrm{dBm}$ IIP3 and $<6.5 \mathrm{~dB}$ NF," in IEEE ISSCC Dig. Tech. Papers, Feb. 2009, pp. $222-223,223 a$

[14] A. N. Bhat, R. A. R. van der Zee, S. Finocchiaro, F. Dantoni, and B. Nauta, "A baseband-matching-resistor noise-canceling receiver architecture to increase in-band linearity achieving $175 \mathrm{MHz}$ TIA bandwidth with a 3-stage inverter-only opamp," in IEEE Radio Freq. Integr. Circuits Symp., Jun. 2019, pp. 155-158.

[15] H. K. Subramaniyan, E. Klumperink, V. Srinivasan, A. Kiaei, and B. Nauta, "RF transconductor linearization robust to process, voltage and temperature variations," IEEE J. Solid-State Circuits, vol. 50, pp. 2591-2602, Nov. 2015.

[16] D. Yang, C. Andrews, and A. Molnar, "Optimized design of N-phase passive mixer-first receivers in wideband operation," IEEE Trans. Circuits Syst. I, Reg. Papers, vol. 62, pp. 2759-2770, Nov. 2015.

[17] C. Andrews and A. C. Molnar, "Implication of passive mixer transperancy for impedance matching and noise figure in passive mixer-first receivers," IEEE Trans. Circuits Syst. I, Reg. Papers, vol. 57, pp. 3092-3103, Dec. 2010.

[18] W. Sansen, "Distortion in elementary transistor circuits," IEEE Trans. Circuits Syst. II, Analog Digit. Signal Process., vol. 46, pp. 315-325, Mar. 1999.

[19] H. Zhang and E. Sanchez-Sinencio, "Linearization tecnhiques for CMOS low noise amplifiers: a tutorial," IEEE Trans. Circuits Syst. I, Reg. Papers, vol. 58, pp. 22-36, Jan. 2011.

[20] M. Soer, E. Klumperink, D. Broek, B. Nauta, and F. Vliet, "Beamformer with constant $G_{m}$ vector modulators and its spatial intermodulation distortion," IEEE J. Solid-State Circuits, vol. 52, pp. 735-746, Mar 2017.

[21] G. Pini, D. Manstretta, and R. Castello, "Analysis and design of a 20-MHz bandwidth, 50.5-dBm OoB-IIP3, and 5.4-mW TIA for SAW-less receivers," IEEE J. Solid-State Circuits, vol. 53, pp. 1468-1480, May 2018.

[22] H. Jung, D. R. Utomo, S. Han, J. Kim, and S. Lee, "An 80 MHZ bandwidth and $26.8 \mathrm{dBm}$ OOB IIP3 transimpendance amplifier with improved nested feedforward compensation and multi-order filtering," IEEE Trans. Circuits Syst. I, Reg. Papers, vol. 67, pp. 3410-3421, Oct. 2020.

[23] B. Nauta, "A CMOS transconductance-C filter technique for very high frequencies," IEEE J. Solid-State Circuits, vol. 27, pp. 142-153, Feb. 1992.

[24] S. D. Willingham, K. W. Martin, and A. Ganesan, "A BiCMOS low-distortion 8-MHz low-pass filter," IEEE J. Solid-State Circuits, vol. 28, pp. 1234-1245, Dec. 1993.
[25] L. Duipmans, R. E. Struiksma, E. A. M. Klumperink, B. Nauta, and F. E. van Vliet, "Analysis of the signal transfer and folding in N-path filters with a series inductance," IEEE Trans. Circuits Syst. I, Reg. Papers, vol. 62, pp. 263-272, Jan. 2015.

[26] S. Pavan and E. Klumperink, "Analysis of the effect of source capacitance and inductance on N-path mixers and filters," IEEE Trans. Circuits Syst. I, Reg. Papers, vol. 65, pp. 1469-1480, May 2018.

[27] S. Jayasuriya, D. Yang, and A. Molnar, "A baseband technique for automated LO leakage suppression achieving $<-80 \mathrm{dbm}$ in wideband passive mixer-first receivers," in IEEE CICC, 2014, pp. 1-4.

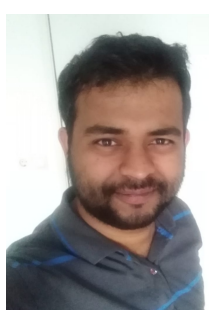

Anoop Narayan Bhat (M'17) received the B.E. degree from the R. V. College of Engineering, Bengaluru, India, in 2012, and the M.S. degree in electrical engineering from IIT Madras, Chennai, India, in 2016. He is currently pursuing the Ph.D. degree with the University of Twente, Enschede, The Netherlands. He was a Design Engineer with Texas Instruments India Ltd., Bengaluru, from 2015 to 2017

He was a recepient of the 2016 Technoinventor award in the masters category from the India Electronics and Semiconductor Association and one of the winners of the 2019 IEEE Solid-State Circuits Society International Student Circuit Contest.

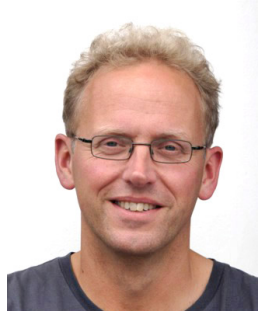

Ronan A.R. van der Zee (M'07) received the M.Sc. (cum laude) degree in electrical engineering and the Ph.D. degree in high efficiency audio amplifiers from the University of Twente, Enschede, The Netherlands, in 1994 and 1999, respectively. In 1999, he joined Philips Semiconductors, where he worked on class $\mathrm{AB}$ and class $\mathrm{D}$ audio amplifiers. In 2003, he joined the IC-Design Group, University of Twente. His research interests include linear and switching power amplifiers, RF frontends and ultralow power radio. 


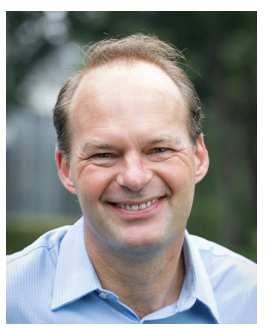

Bram Nauta (M'91-SM'03-F'07) was born in 1964 in Hengelo, The Netherlands. In 1987 he received the M.Sc degree (cum laude) in electrical engineering from the University of Twente, Enschede, The Netherlands. In 1991 he received the $\mathrm{Ph} . \mathrm{D}$. degree from the same university on the subject of analog CMOS filters for very high frequencies. In 1991 he joined the Mixed-Signal Circuits and Systems Department of Philips Research, Eindhoven the Netherlands. In 1998 he returned to the University of Twente, where he is currently a distinguished professor, heading the IC Design group. Since 2016 he also serves as chair of the EE department at this university. His current research interest is high-speed analog CMOS circuits, software defined radio, cognitive radio and beamforming.

He served as the Editor-in-Chief (2007-2010) of the IEEE Journal of Solid-State Circuits (JSSC), and was the 2013 program chair of the International Solid State Circuits Conference (ISSCC). He served as the President of the IEEE Solid-State Circuits Society (2018-2019 term).

Also, he served as Associate Editor of IEEE Transactions on Circuits and Systems II (1997-1999), and of JSSC (2001-2006). He was in the Technical Program Committee of the Symposium on VLSI circuits (2009-2013) and is in the steering committee and programme committee of the European Solid State Circuit Conference (ESSCIRC). He served as distinguished lecturer of the IEEE, is co-recipient of the ISSCC 2002 and 2009 "Van Vessem Outstanding Paper Award" and in 2014 he received the 'Simon Stevin Meester' award $(500.000 €)$, the largest Dutch national prize for achievements in technical sciences. He is fellow of the IEEE and member of the Royal Netherlands Academy of Arts and Sciences (KNAW). 